\title{
Flat-band physics in the spin-1/2 sawtooth chain
}

\author{
Oleg Derzhko ${ }^{1,2, a}$, Jürgen Schnack $^{3}$, Dmitry V. Dmitriev ${ }^{4}$, Valery Ya. Krivnov ${ }^{4}$, and Johannes Richter ${ }^{2,5}$ \\ ${ }^{1}$ Institute for Condensed Matter Physics, National Academy of Sciences of Ukraine, Svientsitskii Street 1, \\ 79011 L'viv, Ukraine \\ ${ }^{2}$ Max-Planck-Institut für Physik komplexer Systeme, Nöthnitzer Straße 38, 01187 Dresden, Germany \\ ${ }^{3}$ Fakultät für Physik, Universität Bielefeld, Postfach 100131, 33501 Bielefeld, Germany \\ ${ }^{4}$ Institute of Biochemical Physics of RAS, Kosygin Street 4, 119334 Moscow, Russia \\ ${ }^{5}$ Institut für Physik, Otto-von-Guericke-Universität Magdeburg, P.O. Box 4120, 39016 Magdeburg, Germany
}

Received 30 April 2020 / Received in final form 5 July 2020

Published online 24 August 2020

(C) The Author(s) 2020. This article is published with open access at Springerlink.com

\begin{abstract}
We consider the strongly anisotropic spin-1/2 $X X Z$ model on the sawtooth-chain lattice with ferromagnetic longitudinal interaction $J^{z z}=\Delta J$ and aniferromagnetic transversal interaction $J^{x x}=J^{y y}=$ $J>0$. At $\Delta=-1 / 2$ the lowest one-magnon excitation band is dispersionless (flat) leading to a massively degenerate set of ground states. Interestingly, this model admits a three-coloring representation of the ground-state manifold [H.J. Changlani et al., Phys. Rev. Lett. 120, 117202 (2018)]. We characterize this ground-state manifold and elaborate the low-temperature thermodynamics of the system. We illustrate the manifestation of the flat-band physics of the anisotropic model by comparison with two isotropic flat-band Heisenberg sawtooth chains. Our analytical consideration is complemented by exact diagonalization and finite-temperature Lanczos method calculations.
\end{abstract}

\section{Introduction}

Frustrated quantum Heisenberg spin systems are of great interest nowadays. Exact calculations and rigorous statements, although scarce, are obviously important for this field. One source of such results stems from the flat-band antiferromagnets, i.e., the models with a dispersionless (flat) one-magnon band [1]. The flat one-magnon band leads to localized multi-magnon states which dominate the low-temperature physics in antiferromagnetic flat-band models close to the saturation field. Their contribution to the partition function can be exactly calculated by visualizing the localized multi-magnon states as hardcore-object configurations on a corresponding auxiliary lattice. Then the hard-core description allows to use classical statistical mechanics to describe frustrated quantum spin models. This approach has been successfully used for a wide class of frustrated quantum antiferromagnets supporting flat bands [2-7] including the kagome antiferromagnet in two dimensions and the pyrochlore antiferromagnet in three dimensions. We mention that a similar description of flat-band states can be developed for the Hubbard model [1,8-12]. A popular one-dimensional example of a flat-band antiferromagnet is the Heisenberg sawtooth chain with the special relation between antiferromagnetic exchange interaction along the basal line $J_{1}>0$ and along the zig-zag path $J_{2}>0$, of $J_{2} / J_{1}=2$, that

\footnotetext{
${ }^{\mathrm{a}}$ e-mail: derzhko@icmp.lviv.ua
}

was widely used as a playground for localized-magnon physics at low temperatures around the saturation field $h_{\text {sat }}=4 J_{1}$, see, e.g., [2-5,13-15].

Later on it was found that flat-band physics and corresponding localized multi-magnon states can appear in frustrated magnets also at zero magnetic field in case that ferro- and antiferromagnetic interactions compete [16]. Again, the sawtooth chain is a prominent example, however, with ferromagnetic bonds $J_{2}<0$ along the zig-zag path and antiferromagnetic bonds $J_{1}>0$ along the basal line $[16-22]$. Here the flat-band physics is realized at a critical point $J_{2} / J_{1}=-2$, where the ferromagnetic ground state gives way for a ferrimagnetic one. It is worth mentioning that the ferro-antiferromagnetic sawtooth chain is an appropriate model to describe the recently synthesized compound $\mathrm{Fe}_{10} \mathrm{Gd}_{10}$ [23] and is also relevant for $\mathrm{Cs}_{2} \mathrm{LiTi}_{3} \mathrm{~F}_{12}$ that hosts ferro-antiferromagnetic sawtooth chains as magnetic subsystems [24].

Very recently, using the three-coloring description Changlani et al. [25,26] have noticed that the groundstate manifold of the spin- $1 / 2 X X Z$ sawtooth chain with antiferromagnetic bonds $J_{1}=J_{2}>0$ and with a negative $z z$ anisotropy parameter $\Delta=-1 / 2$ (denoted as $X X Z 0$ model) exhibits also a huge degeneracy. As already noticed before (but not investigated) in reference [19], the $X X Z 0$ sawtooth chain also belongs to the class of flat-band systems hosting localized multi-magnon states in zero magnetic field. The three-coloring description of spin systems is a general and promising approach to 

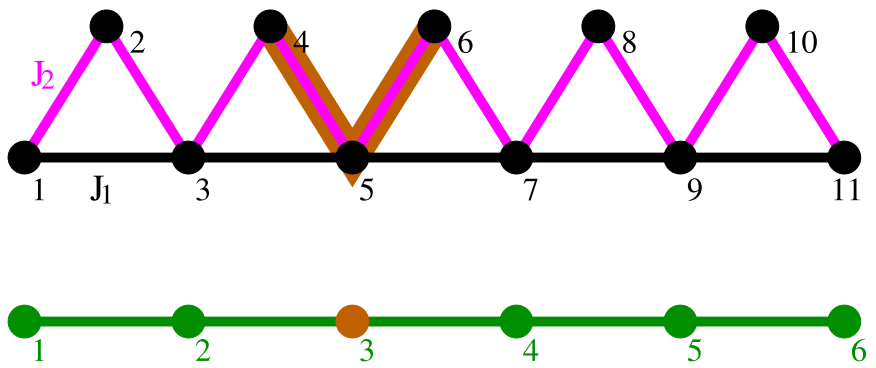

Fig. 1. (Top) The sawtooth-chain lattice (here, $N=11$ sites and open boundary conditions are imposed) considered in the present study. The brownly highlighted bonds mark a trap for a localized magnon on the sawtooth chain, see equations (6)(9). (Bottom) Auxiliary simple chain used for characterization of the ground states of the sawtooth-chain spin models. A localized magnon is represented by a brown site of the simple chain.

study frustrated magnets [25-29]. To illustrate the relation between the three-coloring and the flat-band localizedmagnon description by the example of the sawtooth spin chain is one of the aims of the present study.

In the present paper, we examine the spin- $1 / 2 X X Z 0$ sawtooth-chain model [25] focusing on the specific flatband features, i.e., localized-magnon properties. We also compare this model with the two isotropic Heisenberg sawtooth-chain flat-band cases which were mentioned above and studied previously $[2-5,13,14,16,19-22]$. To be specific, in what follows we consider the spin-1/2 $X X Z$ Hamiltonian

$$
\begin{aligned}
H= & J_{1} \sum_{i=1}^{\mathcal{N}}\left(s_{2 i-1}^{x} s_{2 i+1}^{x}+s_{2 i-1}^{y} s_{2 i+1}^{y}+\Delta_{1} s_{2 i-1}^{z} s_{2 i+1}^{z}\right) \\
& +J_{2} \sum_{i=1}^{\mathcal{N}}\left(s_{2 i-1}^{x} s_{2 i}^{x}+s_{2 i-1}^{y} s_{2 i}^{y}+\Delta_{2} s_{2 i-1}^{z} s_{2 i}^{z}\right. \\
& \left.+s_{2 i}^{x} s_{2 i+1}^{x}+s_{2 i}^{y} s_{2 i+1}^{y}+\Delta_{2} s_{2 i}^{z} s_{2 i+1}^{z}\right)-h \sum_{i=1}^{N} s_{i}^{z}
\end{aligned}
$$

on the sawtooth-chain lattice of $N$ sites (see Fig. 1), where we have $\mathcal{N}=(N-1) / 2$ for open boundary conditions and $\mathcal{N}=N / 2$ for periodic boundary conditions. In what follows we choose the following flat-band parameter sets:

- model 1: $J_{2}=J_{1}>0, \Delta_{1}=\Delta_{2}=-1 / 2[25]$;

- model 2: $J_{2}=-2 J_{1}<0, \Delta_{1}=\Delta_{2}=1[16]$;

- model 3: $J_{2}=2 J_{1}>0, \Delta_{1}=\Delta_{2}=1$ [5],

where model 1 corresponds to the $X X Z 0$ model mentioned above. It is convenient to set $J_{1}=1$ for model 1 [25], but $J_{1}=1 / 2$ for models 2 [16] and 3 .

In addition to analytical investigations of the models presented in Sections 2-4 we will use full exact diagonalization (ED) employing J. Schulenburg's spinpack code [30] and the finite-temperature Lanczos (FTL) technique [31-35] to discuss numerical data for finite sawtooth chains in Sections 4 and 5.

\section{Constituents for many-body physics}

We begin with the illustration of some key elements relevant for the localized-magnon picture and for the three-coloring representation. First of all we note that the spin Hamiltonian $H$ commutes with total $S^{z}=\sum_{i=1}^{N} s_{i}^{z}$ that allows us to consider the eigenstates of the Hamiltonian in each of $N+1$ subspaces of $S^{z}=N / 2, N / 2-$ $1, \ldots,-N / 2$ separately. Clearly, the fully polarized ferromagnetic state $|0\rangle$ is the only eigenstate of $H$ in the subspace with $S^{z}=N / 2$ with the energy $E_{0}$ and it can be considered as the magnon vacuum state. It is straightforward to get the eigenstates and eigenvalues in the subspace with $S^{z}=N / 2-1$ (one-magnon states), see below.

Since the sawtooth chain is a one-dimensional array of corner-sharing triangles, see Figure 1, its Hamiltonian can be written as a sum over Hamiltonians of each triangle,

$$
\begin{aligned}
H= & \sum_{\triangle} H_{\triangle} \\
H_{\triangle}= & J_{1}\left(s_{2 i-1}^{x} s_{2 i+1}^{x}+s_{2 i-1}^{y} s_{2 i+1}^{y}+\Delta_{1} s_{2 i-1}^{z} s_{2 i+1}^{z}\right) \\
& +J_{2}\left(s_{2 i-1}^{x} s_{2 i}^{x}+s_{2 i-1}^{y} s_{2 i}^{y}+\Delta_{2} s_{2 i-1}^{z} s_{2 i}^{z}\right. \\
& \left.+s_{2 i}^{x} s_{2 i+1}^{x}+s_{2 i}^{y} s_{2 i+1}^{y}+\Delta_{2} s_{2 i}^{z} s_{2 i+1}^{z}\right)
\end{aligned}
$$

(the Zeeman term is omitted). Below we also discuss the eigenstates and eigenvalues of the spin Hamiltonian $H_{\triangle}$ as they explain the three-coloring representation for the sawtooth-chain spin model $\mathbf{1}$ and provide a route to construct the eigenstates of the sawtooth-chain spin models $\mathbf{1}$ and $\mathbf{2}$.

It is worth mentioning that the triangles in model $\mathbf{1}$ have the full $C_{3}$ symmetry before they are connected and that models $\mathbf{2}$ and $\mathbf{3}$ are isotropic in spin space.

\subsection{Flat bands and localized magnons}

Imposing periodic boundary conditions, $s_{N+1}^{\alpha}=s_{1}^{\alpha}(N$ is even), we find straightforwardly the energies of the onemagnon excitations above the fully polarized ferromagnetic state $|0\rangle$ (magnon vacuum) for all three spin models. All of them exhibit flat bands. For the models at hand we have

$$
E_{1}(k)-E_{0}=0, \quad E_{2}(k)-E_{0}=\frac{3}{2}+\cos k
$$

for model 1,

$$
E_{1}(k)-E_{0}=0, \quad E_{2}(k)-E_{0}=\frac{3}{2}+\frac{1}{2} \cos k
$$

for model $\mathbf{2}$, and

$$
E_{1}(k)-E_{0}=-2, \quad E_{2}(k)-E_{0}=-\frac{1}{2}+\frac{1}{2} \cos k
$$

for model 3 (recall that $J_{1}=1 / 2$ for models 2 and $\mathbf{3}$ ), where $E_{0}$ is the energy of the ferromagnetic state. Here, as usually, $k$ acquires $N / 2$ values within the region between 
$-\pi$ and $\pi$. Note that in the cases $\mathbf{1}$ and $\mathbf{2}$ the (lowestenergy) flat-band excitations have zero energy.

Furthermore, one can construct the flat-band states as localized states where a magnon is located on three adjacent sites of the lattice (magnon trap), see Figure 1, where a trap built by sites $4,5,6$ is brownly highlighted. We have

$$
\left|l_{i}\right\rangle=l_{i}|0\rangle, \quad i=1, \ldots, \frac{N}{2}
$$

with

$$
l_{i}=s_{2 i-2}^{-}-s_{2 i-1}^{-}+s_{2 i}^{-}
$$

for model $\mathbf{1}$,

$$
l_{i}=s_{2 i-2}^{-}+2 s_{2 i-1}^{-}+s_{2 i}^{-}
$$

for model $\mathbf{2}$, and

$$
l_{i}=s_{2 i-2}^{-}-2 s_{2 i-1}^{-}+s_{2 i}^{-}
$$

for model 3, see Figure 1. Note that with periodic boundary conditions $0 \equiv N$, i.e., $s_{0}^{-} \equiv s_{N}^{-}$. The local nature of the one-magnon ground states (6) allows to construct many-magnon ground states, see Section 3.

\subsection{Spin model on a triangle}

Here we provide some formulas which we need in the following sections. Eight eigenstates of the triangle Hamiltonian $H_{\triangle}(2)$ with different $S^{z}=3 / 2,1 / 2,-1 / 2,-3 / 2$ for the model 1 may be written in the form

$$
\begin{aligned}
|1\rangle & =|\uparrow \uparrow \uparrow\rangle, \\
\left|2_{\chi}\right\rangle & =|\downarrow \uparrow \uparrow\rangle+\omega|\uparrow \downarrow \uparrow\rangle+\omega^{2}|\uparrow \uparrow \uparrow \downarrow\rangle, \\
\left|3_{\chi}\right\rangle & =|\downarrow \uparrow \uparrow\rangle+\omega^{2}|\uparrow \downarrow \uparrow\rangle+\omega \mid \uparrow \uparrow \uparrow \downarrow, \\
|4\rangle & =|\downarrow \uparrow \uparrow\rangle+|\uparrow \downarrow \uparrow\rangle+|\uparrow \uparrow \downarrow\rangle, \\
\left|5_{\chi}\right\rangle & =|\uparrow \downarrow \downarrow\rangle+\omega|\downarrow \uparrow \downarrow\rangle+\omega^{2}|\downarrow \downarrow \uparrow\rangle, \\
\left|6_{\chi}\right\rangle & =|\uparrow \downarrow \downarrow\rangle+\omega^{2}|\downarrow \uparrow \downarrow\rangle+\omega|\downarrow \downarrow \uparrow\rangle, \\
|7\rangle & =|\uparrow \downarrow \downarrow\rangle+|\downarrow \uparrow \downarrow\rangle+|\downarrow \downarrow \uparrow\rangle, \\
|8\rangle & =|\downarrow \downarrow \downarrow\rangle,
\end{aligned}
$$

where the states $|1\rangle,\left|2_{\chi}\right\rangle,\left|3_{\chi}\right\rangle,\left|5_{\chi}\right\rangle,\left|6_{\chi}\right\rangle$, and $|8\rangle$ are the ground states of the triangle with the eigenvalue $-3 / 8$ and the states $|4\rangle$ and $|7\rangle$ are excited states with the eigenvalue $9 / 8$. Here

$$
\omega=\exp \frac{2 \pi \mathrm{i}}{3} .
$$

The eigenstates $\left|2_{\chi}\right\rangle,\left|3_{\chi}\right\rangle,\left|5_{\chi}\right\rangle$, and $\left|6_{\chi}\right\rangle$, are also the eigenstates of the chirality operator of the triangle and thus their form is important for constructing a threecoloring representation for the ground-state manifold of model 1 [25]. However, to get a better relation to the investigations of references $[16,19]$ we may introduce other linear combinations of these states, namely,

$$
\begin{aligned}
& |2\rangle=|\downarrow \uparrow \uparrow\rangle-|\uparrow \downarrow \uparrow\rangle \propto\left|2_{\chi}\right\rangle-\omega\left|3_{\chi}\right\rangle, \\
& |3\rangle=|\downarrow \uparrow \uparrow\rangle-|\uparrow \uparrow \downarrow\rangle \propto \omega\left|2_{\chi}\right\rangle-\left|3_{\chi}\right\rangle, \\
& |5\rangle=|\uparrow \downarrow \downarrow\rangle-|\downarrow \uparrow \downarrow\rangle \propto\left|5_{\chi}\right\rangle-\omega\left|6_{\chi}\right\rangle, \\
& |6\rangle=|\uparrow \downarrow \downarrow\rangle-|\downarrow \downarrow \uparrow\rangle \propto \omega\left|5_{\chi}\right\rangle-\left|6_{\chi}\right\rangle .
\end{aligned}
$$

We use these eigenstates in Section 3 while constructing many-magnon ground states.

For the model $\mathbf{2}$, the states $|\uparrow \uparrow \uparrow\rangle,|\downarrow \uparrow \uparrow\rangle+|\uparrow \downarrow \uparrow\rangle+|\uparrow \uparrow \downarrow\rangle$, $|\downarrow \uparrow \uparrow\rangle-|\uparrow \uparrow \downarrow\rangle,|\uparrow \downarrow \downarrow\rangle+|\downarrow \uparrow \downarrow\rangle+|\downarrow \downarrow \uparrow\rangle,|\uparrow \downarrow \downarrow\rangle-|\downarrow \downarrow \uparrow\rangle$, $|\downarrow \downarrow \downarrow\rangle$ are the eigenstates with the eigenvalue $-3 / 8$ and the states $|\downarrow \uparrow \uparrow\rangle-2|\uparrow \downarrow \uparrow\rangle+|\uparrow \uparrow \downarrow\rangle,|\uparrow \downarrow \downarrow\rangle-2|\downarrow \uparrow \downarrow\rangle+|\downarrow \downarrow \uparrow\rangle$ are the eigenstates with the eigenvalue $9 / 8$.

For consistency, we give also the states for the model 3. This set of states has a completely different structure: The highest-energy is the quadruplet $(|\uparrow \uparrow \uparrow\rangle,|\downarrow \uparrow \uparrow\rangle+\mid \uparrow \downarrow \uparrow$ \rangle$+|\uparrow \uparrow \downarrow\rangle$ etc.) with the energy $5 / 8$, the two states $\mid \downarrow \uparrow \uparrow$ \rangle$-|\uparrow \uparrow \downarrow\rangle$ and $|\uparrow \downarrow \downarrow\rangle-|\downarrow \downarrow \uparrow\rangle$ have the energy $-3 / 8$, and finally the two states $|\downarrow \uparrow \uparrow\rangle-2|\uparrow \downarrow \uparrow\rangle+|\uparrow \uparrow \downarrow\rangle$ and $\mid \uparrow \downarrow \downarrow$ \rangle$-2|\downarrow \uparrow \downarrow\rangle+|\downarrow \downarrow \uparrow\rangle$ have the energy $-7 / 8$.

\section{Many-magnon states of model 1}

So far only for the models $\mathbf{2}$ and $\mathbf{3}$ the construction of localized-magnon states was described in the literature, see, e.g., reference [16] for model $\mathbf{2}$ and references [2,3,13] for model 3, but not for model $\mathbf{1}$. Therefore, we sketch now the construction rules of localized-magnon states for model 1 in this section. In addition, we will briefly discuss the relation of the localized-magnon states to the promising three-coloring picture developed in references [25,26].

We begin with a short outline of the three-coloring representation for the ground-state manifold of the spin- $1 / 2$ $X X Z 0$ sawtooth chain (i.e., model 1 in our notation). The starting point is the definition of three single-spin coloring states

$$
|r\rangle \equiv|\uparrow\rangle+|\downarrow\rangle,|b\rangle \equiv|\uparrow\rangle+\omega|\downarrow\rangle,|g\rangle \equiv|\uparrow\rangle+\omega^{2}|\downarrow\rangle,
$$

where $\omega=\exp (2 \pi \mathrm{i} / 3)$, see equation (11). The states in equation (13) describe spin- $1 / 2$ coherent states [36], associated with three particular directions, each separated by 120 degrees, in the plane perpendicular to the $z$-axis. These states are represented by the colors red, blue, green, respectively. A multi-spin state on a lattice is constructed by putting a single-spin coloring state at each lattice site. The state can be graphically represented as a threecoloring of the lattice (i.e., no two vertices connected by a bond have the same color). Obviously, the two threecoloring states $\left|r_{1} b_{2} g_{3}\right\rangle$ and $\left|r_{1} g_{2} b_{3}\right\rangle$ on a triangle are superpositions of states given in equation (10) with the ground-state energy $-3 / 8$, where states with different $S^{z}$ are mixed. In other words, the states $\left|r_{1} b_{2} g_{3}\right\rangle$ and $\left|r_{1} g_{2} b_{3}\right\rangle$ belong to the ground-state manifold. The three-coloring can be straightforwardly extended to a thermodynamically large lattice. The total number of three-colorings for the open sawtooth chain of $N=2 \mathcal{N}+1$ sites grows as $2^{\mathcal{N}}$, where $\mathcal{N}=(N-1) / 2$ is the number of triangles in 
the open sawtooth chain. Moreover, constructing resonating color loops, one can single out a localized magnon state [26], see also below for an example. However, by contrast to the flat-band localized-magnon description, the utilization of the three-coloring picture to determine properties of corresponding frustrated spin models, such as model $\mathbf{1}$, is much less elaborated, i.e., it is still a task to be addressed in the future. One difficulty is certainly the mixing of states with different $S^{z}$ that requires a subsequent projection onto the $S^{z}$-subspaces to restore this symmetry of Hamiltonian (1). In what follows, we therefore mainly exploit the localized-magnon picture for the one-dimensional sawtooth-chain spin model.

First we mention that in the subspace $S^{z}=N / 2-1$ the localized-magnon (or flat-band) states introduced in Section 2 are exact eigenstates. For the sawtooth chain model 1 of $N=2 \mathcal{N}+1$ sites with open boundary conditions there are two classes of localized one-magnon states, namely, "boundary" states such as

$$
\left|l_{1}\right\rangle=\left(-\left|\downarrow_{1} \uparrow_{2} \uparrow_{3}\right\rangle+\left|\uparrow_{1} \downarrow_{2} \uparrow_{3}\right\rangle\right)|\ldots \uparrow \ldots\rangle
$$

and "bulk" states such as

$$
\left|l_{2}\right\rangle=\left(\left|\downarrow_{2} \uparrow_{3} \uparrow_{4}\right\rangle-\left|\uparrow_{2} \downarrow_{3} \uparrow_{4}\right\rangle+\left|\uparrow_{2} \uparrow_{3} \downarrow_{4}\right\rangle\right)|\ldots \uparrow \ldots\rangle,
$$

where the numbers at the up- and down-arrows correspond to the numbering in Figure 1, top; $|\ldots \uparrow \ldots\rangle$ in equation (15) means the state with all the spins $1,5,6, \ldots$ up. Both states belong to the ground-state manifold. (For an explicit proof we refer to Appendix A.) Note here that the localized boundary ground states exist also for model $\mathbf{2}^{1}$ but not for model 3 .

Let us also give an example how to get a localized magnon state from the three-coloring representation. We have

$$
\left|l_{2}\right\rangle \propto P_{S^{z}=\frac{N}{2}-1}\left(\left|r_{1} b_{2} g_{3} b_{4} r_{5} \ldots\right\rangle-\left|r_{1} g_{2} b_{3} g_{4} r_{5} \ldots\right\rangle\right),
$$

where $P_{S^{z}}$ stands for the projector onto the subspace with the specific $S^{z}$ and the numbers $1, \ldots, 5$ correspond to those given in the first line of Figure 1.

In summary, the ground-state degeneracy for the open sawtooth chain 1 of $N=2 \mathcal{N}+1$ sites in the subspace $S^{z}=N / 2-1$ is $\mathcal{N}+1$, because all the localized-magnon states are linearly independent [37]. A corresponding consideration holds for the open sawtooth-chain model $\mathbf{2}$, i.e., the ground-state degeneracy is also $\mathcal{N}+1$. On the other hand, for the open sawtooth-chain model 3 the degeneracy in the subspace $S^{z}=N / 2-1$ is lower and equals $\mathcal{N}-1$, because the localized boundary states are missing.

We pass to the subspace $S^{z}=N / 2-k$ with $k=2$ down spins. Because of the localized nature of the onemagnon excitations, independent localized two-magnon eigenstates can be constructed satisfying the hard-dimer rule (see lines 1 and 2 in Fig. 2), i.e., two localized onemagnon states are not allowed to be in touch. There are $\mathcal{C}_{\mathcal{N}-k+2}^{k}, k=2$, such states for the open sawtooth

\footnotetext{
${ }^{1}$ The analogue of the state (14) for the model 2 is $\left|l_{1}\right\rangle=$ $\left(2\left|\downarrow_{1} \uparrow_{2} \uparrow_{3}\right\rangle+\left|\uparrow_{1} \downarrow_{2} \uparrow_{3}\right\rangle\right)\left|\ldots \uparrow_{\ldots}\right\rangle$.
}

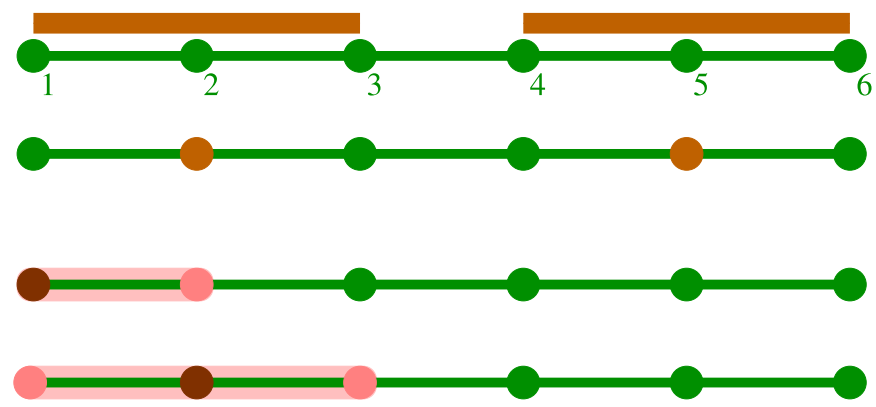

Fig. 2. Visualization of the ground states in the subspace $S^{z}=N / 2-2$ on the auxiliary linear chain (open boundary conditions) which corresponds to the $N=11$ sawtooth chain. Line 1: two independent localized magnons can be pictorially represented as a spatial configuration of two hard dimers. A hard dimer extends over two lattice constants. Overlapping of two dimers is forbidden, i.e., two neighboring sites of the auxiliary linear chain cannot be occupied by independent localized magnons. Line 2: corresponding position of the localized magnons (filled brown circles). Lines 3 and 4: two different two-magnon complexes of overlapping localized magnons corresponding to equations (17) (line 3) (18) (line 4).

chain of $N=2 \mathcal{N}+1$ sites, where $\mathcal{C}_{m}^{n}=m ! /[n !(m-n) !]$ is the binomial coefficient. This construction rule was first found for model 3 and can be extended to more than two magnons (so called independent localized multi-magnon states) leading finally to a huge ground-state degeneracy of model 3 at the saturation field $h_{\text {sat }}$ that grows exponentially with system size $N$, cf. $[2,3,13]$. Obviously, the above illustrated construction of independent localized multi-magnon states also holds for models $\mathbf{1}$ and 2 [16,19]; the (natural) number of the magnons $k$ for these open chains varies in the region $2 \leq k \leq(\mathcal{N}+2) / 2$. However, there are two important differences to model 3: (i) all these multi-magnon states are degenerate at zero field $h=0$ [cf. Eqs. (3)-(5)] and (ii) in addition to the independent localized-magnon states also specifically overlapping localized magnons are ground states [16]. Thus, the ground-state degeneracy of models $\mathbf{1}$ and $\mathbf{2}$ is much larger than for model 3.

To illustrate overlapping localized magnons, we consider a localized two-magnon complex at the boundary defined as $[16,19]$

$$
\begin{aligned}
l_{1}\left(c l_{1}+l_{2}\right)|0\rangle & =l_{1}\left(-\frac{l_{1}}{2}+l_{2}\right)|0\rangle \\
& =\left(-s_{1}^{-}+s_{2}^{-}\right)\left(\frac{s_{1}^{-}}{2}+\frac{s_{2}^{-}}{2}-s_{3}^{-}+s_{4}^{-}\right)|0\rangle,
\end{aligned}
$$

where in equation (17) we have defined the symbol $c=$ $-1 / 2$ instead of using the concrete value $-1 / 2$ to avoid cumbersome expressions in a number of formulas given below, see line 3 in Figure 2 for a pictorial representation of the state (17). In Appendix A we check that this state is among the ground states with $S^{z}=N / 2-2$. 

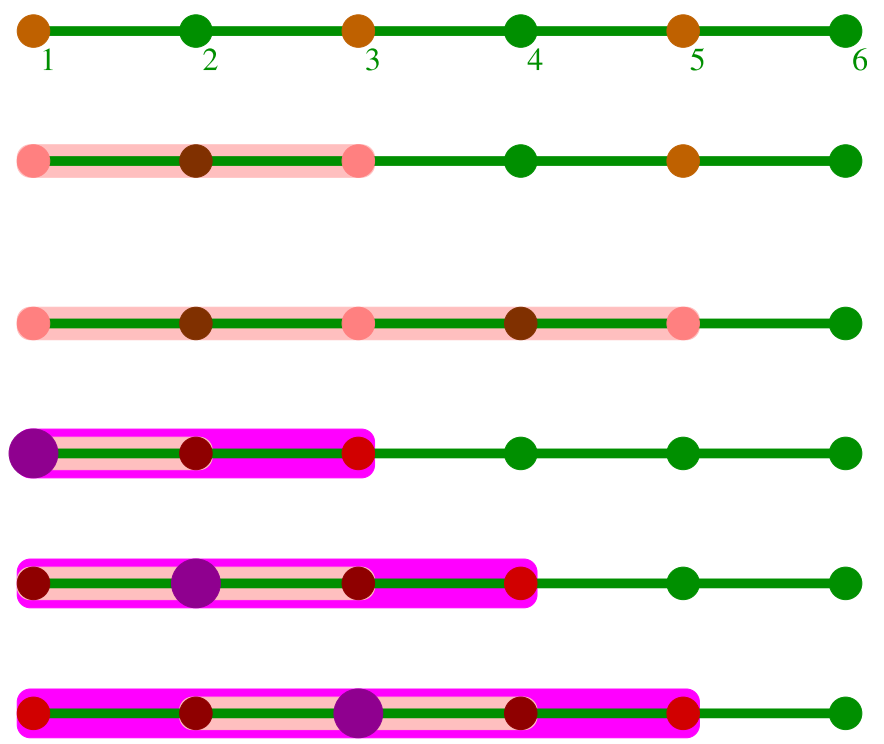

Fig. 3. Visualization of the ground states in the subspace $S^{z}=N / 2-3$ (here $N=11$ sites, open boundary conditions). Line 1: three independent localized magnons can be pictorially represented as a spatial configuration of three hard dimers. Line 2: localized magnon and localized two-magnon complex. Line 3: localized three-magnon complex of one-bracket type, see equation (20). Lines 4, 5, and 6: localized three-magnon complexes of two-bracket type, see equation (22).

A two-magnon complex away from the boundary is given by the formula

$$
\begin{aligned}
& l_{2}\left(l_{1}+c l_{2}+l_{3}\right)|0\rangle=l_{2}\left(l_{1}-\frac{l_{2}}{2}+l_{3}\right)|0\rangle \\
& \quad=\left(s_{2}^{-}-s_{3}^{-}+s_{4}^{-}\right)\left(-s_{1}^{-}+\frac{s_{2}^{-}}{2}+\frac{s_{3}^{-}}{2}+\frac{s_{4}^{-}}{2}-s_{5}^{-}+s_{6}^{-}\right)|0\rangle
\end{aligned}
$$

$(c=-1 / 2)$, see line 4 in Figure 2. Again, in Appendix A we check that this state is among the ground states with $S^{z}=N / 2-2$.

It is easy to count the ground states in the sector $S^{z}=$ $N / 2-2$. We have $\mathcal{C}_{\mathcal{N}}^{2}$ independent localized two-magnon states and $\mathcal{N}+1$ localized states built by localized twomagnon complexes, in total

$$
g_{\mathcal{N}}\left(S^{z}=N / 2-2\right)=\mathcal{C}_{\mathcal{N}}^{2}+\mathcal{N}+1=\sum_{k=0}^{2} \mathcal{C}_{\mathcal{N}}^{k}
$$

We have checked equation (19) by exact diagonalization of open sawtooth chains $\mathbf{1}$ of up to $N=39$ sites providing evidence for the completeness of the constructed ground states in the subspace $S^{z}=N / 2-2$. Moreover, these states are linearly independent [37].

Now we turn to the subspace $S^{z}=N / 2-k$ with $k=3$ spins down. Again, because of the local nature of the independent localized-magnon states and the localized two-magnon complexes, we can construct a number of ground states in the sector $S^{z}=N / 2-3$ placing such states sufficiently far from each other. This way we construct $\mathcal{C}_{\mathcal{N}-1}^{3}$ independent localized magnon states and $2 \mathcal{C}_{\mathcal{N}-1}^{2}$ states consisting of a localized magnon and a localized two-magnon complex, see lines 1 and 2 in Figure 3 for $N=11$. More ground states in this subspace are the localized three-magnon complexes, where we have two types, which we will denote as one-bracket type and two-bracket type, see equations (20)-(22) below. These localized threemagnon complexes are sketched in Figure 3 for $N=11$, see line 3 for the one-bracket type and lines 4,5 , and 6 for the two-bracket type.

An example of a localized three-magnon complex of onebracket type that belongs to the ground-state manifold with $S^{z}=N / 2-3$, see Appendix A, is given by

$$
l_{2} l_{4}\left(l_{1}+c l_{2}+l_{3}+c l_{4}+l_{5}\right)|0\rangle
$$

$(c=-1 / 2)$, see line 3 in Figure 3. Other three-magnon complexes of one-bracket type are given by the formulas:

$$
\begin{array}{r}
l_{1} l_{3}\left(c l_{1}+l_{2}+c l_{3}+l_{4}\right)|0\rangle, \\
l_{3} l_{5}\left(l_{2}+c l_{3}+l_{4}+c l_{5}+l_{6}\right)|0\rangle, \\
\vdots \\
l_{\mathcal{N}-1} l_{\mathcal{N}+1}\left(l_{\mathcal{N}-2}+c l_{\mathcal{N}-1}+l_{\mathcal{N}}+c l_{\mathcal{N}+1}\right)|0\rangle .
\end{array}
$$

Altogether, there are $\mathcal{N}-1$ three-magnon complexes of one-bracket type.

We pass to the $\mathcal{N}+1$ localized three-magnon complexes of two-bracket type

$$
\begin{array}{r}
l_{1}\left(c l_{1}+l_{2}\right)\left(c^{2} l_{1}+c l_{2}+l_{3}\right)|0\rangle+d l_{1}^{3}|0\rangle, \\
l_{2}\left(l_{1}+c l_{2}+l_{3}\right)\left(c l_{1}+c^{2} l_{2}+c l_{3}+l_{4}\right)|0\rangle+d l_{2}^{3}|0\rangle, \\
l_{3}\left(l_{2}+c l_{3}+l_{4}\right)\left(l_{1}+c l_{2}+c^{2} l_{3}+c l_{4}+l_{5}\right)|0\rangle+d l_{3}^{3}|0\rangle, \\
\vdots \\
l_{\mathcal{N}+1}\left(l_{\mathcal{N}}+c l_{\mathcal{N}+1}\right)\left(l_{\mathcal{N}-1}+c l_{\mathcal{N}}+c^{2} l_{\mathcal{N}+1}\right)|0\rangle+d l_{\mathcal{N}+1}^{3}|0\rangle .
\end{array}
$$

$(c=-1 / 2$ and $d=1 / 8)$, see lines 4,5 , and 6 in Figure 3. (Note that the last term in the first and the last lines of equation (22) is redundant [since $l_{1}^{3}=\left(-s_{1}^{-}+s_{2}^{-}\right)^{3}=0$ and $\left.l_{\mathcal{N}+1}^{3}=\left(s_{\mathcal{N}}^{-}-s_{\mathcal{N}+1}^{-}\right)^{3}=0\right]$, it is written for similarity to the other lines, where this kind of terms are relevant.) Again, some more detailed calculations checking that the above presented states are ground states with $S^{z}=$ $N / 2-3$ are transferred to Appendix A.

In sum, the number of ground states in the sector $S^{z}=$ $N / 2-3$ is

$$
\begin{aligned}
g_{\mathcal{N}}\left(S^{z}=N / 2-3\right) & =\mathcal{C}_{\mathcal{N}-1}^{3}+2 \mathcal{C}_{\mathcal{N}-1}^{2}+\mathcal{N}-1+\mathcal{N}+1 \\
& =\sum_{k=0}^{3} \mathcal{C}_{\mathcal{N}}^{k}
\end{aligned}
$$

As previously, we have confirmed the analytical expression (23) by exact diagonalization for models $\mathbf{1}$ with open boundary conditions of up to $N=39$ sites. Moreover, these states are linearly independent. 
The construction of the linearly independent ground states for $k \geq 4$ follows the same lines as explained above, although it becomes more tedious. We find that model $\mathbf{1}$ with open boundary conditions is identical to model $\mathbf{2}$ [16]. After all, the degeneracy of the ground-state manifold of the open sawtooth-chain model 1 with $N=2 \mathcal{N}+1$ sites in the subspaces $S^{z}=N / 2-k$ for $k=0,1,2, \ldots, \mathcal{N}$ is given by

$$
g_{\mathcal{N}}\left(S^{z}=N / 2-k\right)=\mathcal{C}_{\mathcal{N}}^{0}+\mathcal{C}_{\mathcal{N}}^{1}+\ldots+\mathcal{C}_{\mathcal{N}}^{k},
$$

cf. equations (19) and (23). Then the total degeneracy of the ground-state manifold of the open sawtooth-chain model $\mathbf{1}$ with $N=2 \mathcal{N}+1$ sites is

$$
\begin{aligned}
\mathcal{W}(N) & =2 \sum_{k=0}^{\mathcal{N}}\left(\mathcal{C}_{\mathcal{N}}^{0}+\mathcal{C}_{\mathcal{N}}^{1}+\ldots+\mathcal{C}_{\mathcal{N}}^{k}\right)=2 \sum_{k=0}^{\mathcal{N}}(\mathcal{N}+1-k) \mathcal{C}_{\mathcal{N}}^{k} \\
& =2\left[(\mathcal{N}+1) 2^{\mathcal{N}}-\mathcal{N} 2^{\mathcal{N}-1}\right]=(\mathcal{N}+2) 2^{\mathcal{N}}
\end{aligned}
$$

As already found for the sectors with $k=2$ and 3 , the general formula (24) and of course also (25) match perfectly with corresponding exact-diagonalization data.

We add here the known information on the degeneracy of the ground-state manifold in the subspaces $S^{z}=N / 2-k$ for $k=0,1,2, \ldots, \mathcal{N}$ of the models 2 and 3 with $N=2 \mathcal{N}+1$ sites and open boundary conditions. For model 2 equation (24) holds [16] and for model 3 the flat-band states exist only in the subspaces $S^{z}=N / 2-k, k=0,1,2, \ldots, \mathcal{N} / 2$, and the degeneracy is $g_{\mathcal{N}}\left(S^{z}=N / 2-k\right)=\mathcal{C}_{\mathcal{N}-k}^{k}$, i.e., it is smaller, because only independent localized multi-magnon states exist, but no additional complexes $[2,3,5]$.

Let us now briefly discuss the ground-state degeneracy of periodic sawtooth chains of $N=2 \mathcal{N}$ sites. For model 3 independent localized multi-magnon ground states exist in the subspace $S^{z}=N / 2-k$ with $k=0,1,2, \ldots, \mathcal{N} / 2$. Their degeneracy is $G_{\mathcal{N}}^{(3)}\left(S^{z}\right)=[\mathcal{N} /(\mathcal{N}-k)] \mathcal{C}_{\mathcal{N}-k}^{k}[2,3,5]$.

For model 2, the ground states in the subspace $S^{z}=$ $N / 2-k$ with $k=0,1,2, \ldots, \mathcal{N}$ were found in references $[16,19]$; their degeneracy is

$$
G_{\mathcal{N}}^{(2)}\left(S^{z}\right)= \begin{cases}\mathcal{C}_{\mathcal{N}}^{k}, & k=0,1, \ldots, \frac{\mathcal{N}}{2}, \\ \mathcal{C}_{\mathcal{N}}^{\frac{\mathcal{N}}{2}}, & k=\frac{\mathcal{N}}{2}, \ldots, \mathcal{N}-1, \\ \mathcal{C}_{\mathcal{N}}^{\frac{\mathcal{N}}{2}}+1, & k=\mathcal{N}\end{cases}
$$

For periodic chains, the model $\mathbf{1}$ exhibits more ground states than the model $\mathbf{2}$ if $k \geq 3$ :

$$
\begin{aligned}
G_{\mathcal{N}}^{(1)}\left(S^{z}\right) & =G_{\mathcal{N}}^{(2)}\left(S^{z}\right)+G_{\mathcal{N}}^{\text {add }}\left(S^{z}\right), \\
G_{\mathcal{N}}^{\text {add }}\left(S^{z}\right) & = \begin{cases}\mathcal{C}_{\mathcal{N}}^{k-3}, & k=3, \ldots, \frac{\mathcal{N}}{2}, \\
2 \mathcal{C}_{\mathcal{N}}^{\frac{\mathcal{N}}{2}}-3-\mathcal{C}_{\mathcal{N}}^{\mathcal{N}-k-3}, & k=\frac{\mathcal{N}}{2}+1, \ldots, \mathcal{N}-3, \\
2 \mathcal{C}_{\mathcal{N}}^{\frac{\mathcal{N}}{2}}-3, & k=\mathcal{N}-2, \mathcal{N}-1, \mathcal{N}\end{cases}
\end{aligned}
$$

The total degeneracy of the ground-state manifold of the periodic sawtooth-chain model 1 with $N=2 \mathcal{N}$ sites then is

$$
\mathcal{W}(N)=\left(\frac{\mathcal{N}}{3}+1\right) 2^{\mathcal{N}}+\frac{2 \mathcal{N}}{3}+1
$$

cf. equation (25). We confirmed the numbers given in equations (26)-(28) by exact diagonalization for the periodic sawtooth-chain model 1 of up to $N=32$ sites (see also Tab. 1).

Apparently, the ground-state degeneracies depend on the imposed boundary conditions for finite chains, but in the thermodynamic limit $N \rightarrow \infty$ the boundary conditions become irrelevant. Therefore, it is sufficient to consider for the analytical calculations of low-temperature thermodynamic quantities (see the next section) the simpler case of open boundary conditions. However, for the numerical techniques used in Section 5 to study finite systems, periodic boundary conditions are more appropriate, because more symmetries can be used, i.e., longer chains are feasible.

\section{Low-temperature thermodynamics of model 1}

From previous investigations of models $\mathbf{2}$ and $\mathbf{3}$ it is known that the huge manifold of localized flat-band ground states may dominate the low-temperature thermodynamics $[2-5,14,16]$. We may expect that this statement is valid also for model 1. Following this argument, in this section we derive analytical formulas for the field and temperature dependences of magnetization, entropy, specific heat, and susceptibility for model $\mathbf{1}$ taking into account flat-band states only. We compare this low-temperature approach with numerical data taking into account all eigenstates.

We consider the influence of a magnetic field $h$ on the manifold of the localized ground states of the open sawtooth-chain model 1 with $N=2 \mathcal{N}+1$ sites. For $h \neq 0$ only the single fully polarized ferromagnetic state remains the ground state with energy $E_{0}(h)=E_{0}-h N / 2$, and all the other localized flat-band states become excited states. The contribution of all these states to the partition function is determined by their degeneracy $g_{\mathcal{N}}\left(S^{z}\right)$ given in equation (24) and their Zeeman energy $E\left(h, S^{z}\right)=$ $E_{0}-h S^{z}$ :

$$
\begin{aligned}
& Z_{\mathrm{fbs}}(T, h, N)=2 \exp \left(-\frac{E_{0}}{T}\right) \\
& \quad \times \sum_{k=0}^{\mathcal{N}}\left(\mathcal{C}_{\mathcal{N}}^{0}+\mathcal{C}_{\mathcal{N}}^{1}+\ldots+\mathcal{C}_{\mathcal{N}}^{k}\right) \cosh \frac{\left(\mathcal{N}+\frac{1}{2}-k\right) h}{T} \\
& =\exp \left(-\frac{E_{0}}{T}\right) \sum_{k=0}^{\mathcal{N}} \mathcal{C}_{\mathcal{N}}^{k} F_{k}(x, \mathcal{N}), \\
& F_{k}(x, \mathcal{N})=\frac{\sinh [(\mathcal{N}+1-k) x]}{\sinh \frac{x}{2}}, \quad x=\frac{h}{T} .
\end{aligned}
$$

As mentioned already above, this part of the partition function is identical for models $\mathbf{1}$ and $\mathbf{2}$ (but not 
for model 3, where no complexes of overlapping localized magnons exist) and may dominate the low-temperature physics. It yields thermodynamic quantities which depend on $x=h / T$ only. Clearly, the full partition functions of models $\mathbf{1}$ and $\mathbf{2}$ are different because of different excited non-flat-band states which come into play at nonzero temperatures, and we can reveal these differences by an exactdiagonalization analysis of finite chains, see Section 5 . We note that (how it should be) for $h=0, T \rightarrow 0$ the partition function reproduces the total ground-state degeneracy (25), i.e., $Z_{\mathrm{fbs}}(x=0, N)=\mathcal{W}(N) \exp \left(-E_{0} / T\right)$.

The residual ground-state entropy is given by $s=$ $\ln [\mathcal{W}(N)] / N$, i.e., we get in the thermodynamic limit for the residual entropy per spin $s=\ln 2 / 2 \approx 0.346574$. As mentioned above, for $N \rightarrow \infty$ the boundary conditions become irrelevant: Because only the exponential term in equations (25) and (28) is essential, we get the same value for open and periodic chains. Obviously, $s=s(T=0)$ is already half of the maximum entropy for $T \rightarrow \infty$. Note that the residual entropy for model $\mathbf{3}$ at the saturation field is smaller, $s=(1 / 2) \ln [(1+\sqrt{5}) / 2] \approx 0.240606[2,3] .^{2}$ Interestingly, although the residual entropy following from equation (25) resembles that of flat-band systems of the so-called monomer universality class $[3,5]$, the universal magneto-thermodynamics for both systems is different: For monomer flat-band systems the partition function is as in equation $(29)$, however, with $F_{k}(x, \mathcal{N})=\exp (k x)$, see reference [5].

A nonzero residual ground-state entropy leads to efficient magnetic cooling $[5,38,39]$. Importantly, for models $\mathbf{1}$ and $\mathbf{2}$ the residual entropy is present at zero field, i.e., it is relevant for cooling by varying the field around zero $[40,41]$, which is obviously an advantage from the practical point of view compared to model 3.

From the Helmholtz free energy obtained from the partition function (29) by $F_{\mathrm{fbs}}(T, h, N)=-T \ln Z_{\mathrm{fbs}}(T, h, N)$ we get thermodynamic quantities such as magnetization, susceptibility, entropy or specific heat. The magnetization $M=-\partial F / \partial h$ and the susceptibility $X=\partial M / \partial h$ are given by the formulas

$$
M_{\text {fbs }}(x, N)=\frac{\sum_{k=0}^{\mathcal{N}} \mathcal{C}_{\mathcal{N}}^{k} \frac{\partial F_{k}(x, \mathcal{N})}{\partial x}}{\sum_{k=0}^{\mathcal{N}} \mathcal{C}_{\mathcal{N}}^{k} F_{k}(x, \mathcal{N})}
$$

${ }^{2}$ This result follows from the formula for the total number of ground states for the open sawtooth chain $\mathbf{3}$ of $N=2 \mathcal{N}+1$ sites at $h=h_{\text {sat }}$,

$$
\mathcal{W}(N)=\sum_{k=0}^{\left[\frac{\mathcal{N}}{2}\right]} \mathcal{C}_{\mathcal{N}-k}^{k}
$$

which implies the following recurrence relation:

$$
\mathcal{W}(N-4)+\mathcal{W}(N-2)=\mathcal{W}(N)
$$

(to prove it, one has to use the identity $\mathcal{C}_{\mathcal{M}}^{p}+\mathcal{C}_{\mathcal{M}}^{p+1}=\mathcal{C}_{\mathcal{M}+1}^{p+1}$ ) or

$$
\frac{\mathcal{W}(N-4)}{\mathcal{W}(N-2)}+1-\frac{\mathcal{W}(N)}{\mathcal{W}(N-2)}=0
$$

Evidently, we have arrived at a Fibonacci sequence. In the limit $N \rightarrow$ $\infty$, the ratio $\mathcal{W}(N-2) / \mathcal{W}(N)$ tends to $\varphi=(\sqrt{5}-1) / 2$, i.e., in the thermodynamic limit, $\mathcal{W}(N)=\varphi^{-\frac{N}{2}}$ resulting in $s=(1 / 2) \ln [(1+$ $\sqrt{5}) / 2]$. and

$$
\begin{aligned}
& T X_{\text {fbs }}(x, N) \\
& =\frac{\sum_{k=0}^{\mathcal{N}} \mathcal{C}_{\mathcal{N}}^{k} \frac{\partial^{2} F_{k}(x, \mathcal{N})}{\partial x^{2}}-\left[\sum_{k=0}^{\mathcal{N}} \mathcal{C}_{\mathcal{N}}^{k} \frac{\partial F_{k}(x, \mathcal{N})}{\partial x}\right]^{2}}{\left[\sum_{k=0}^{\mathcal{N}} \mathcal{C}_{\mathcal{N}}^{k} F_{k}(x, \mathcal{N})\right]^{2}},
\end{aligned}
$$

respectively. The entropy $S=-\partial F / \partial T$ and the specific heat $C=T \partial S / \partial T$ are given by the formulas

$$
\begin{aligned}
S_{\mathrm{fbs}}(x, N) & =\ln \sum_{k=0}^{\mathcal{N}} \mathcal{C}_{\mathcal{N}}^{k} F_{k}(x, \mathcal{N})-x \frac{\sum_{k=0}^{\mathcal{N}} \mathcal{C}_{\mathcal{N}}^{k} \frac{\partial F_{k}(x, \mathcal{N})}{\partial x}}{\sum_{k=0}^{\mathcal{N}} \mathcal{C}_{\mathcal{N}}^{k} F_{k}(x, \mathcal{N})} \\
& =\ln Z_{\mathrm{fbs}}(x, N)-x M_{\mathrm{fbs}}(x, N)
\end{aligned}
$$

and

$$
\begin{aligned}
C_{\mathrm{fbs}}(x, N) & =x^{2} \frac{\sum_{k=0}^{\mathcal{N}} \mathcal{C}_{\mathcal{N}}^{k} \frac{\partial^{2} F_{k}(x, \mathcal{N})}{\partial x^{2}}-\left[\sum_{k=0}^{\mathcal{N}} \mathcal{C}_{\mathcal{N}}^{k} \frac{\partial F_{k}(x, \mathcal{N})}{\partial x}\right]^{2}}{\left[\sum_{k=0}^{\mathcal{N}} \mathcal{C}_{\mathcal{N}}^{k} F_{k}(x, \mathcal{N})\right]^{2}} \\
& =x^{2} T X_{\mathrm{fbs}}(x, N)
\end{aligned}
$$

respectively. Clearly, the magnetization is an odd function of $x$, whereas the susceptibility, the entropy, and the specific heat are even functions of $x$. Below we consider the thermodynamic quantities per site to be denoted by small letters, e.g., $m=M / N$, etc.

The contribution of the flat-band states to the partition function is identical for models $\mathbf{1}$ and $\mathbf{2}$ in the thermodynamic limit as well as for finite open sawtooth chains. This contribution was discussed in detail in reference [16]. In particular, it was shown that the magnetization $m_{\mathrm{fbs}}(x)$ calculated in such a reduced basis depends essentially on $N$ and in the thermodynamic limit $N \rightarrow \infty$ it tends to $1 / 4$ at $x=h / T \rightarrow 0$ in contradiction to the theorem that the magnetization should vanish in vanishing field at $T>0$ for one-dimensional systems. However, for finite chains the "reduced-set" magnetization given by equation (30) may give a good estimate, see Figure 3 and discussion after equation (31) in reference [16].

To illustrate the contribution of the localized flatband states to the low-temperature thermodynamics in a magnetic field, we compare in Figure 4 thermodynamic quantities as they follow from equation (30)-(33) with exact-diagonalization data for the full open sawtoothchain model $1\left(J_{1}=1\right)$ with $N=19$. While for $T=0.001$ the results are indistinguishable (top panel), for $T=0.05$ the difference is definitely seen (bottom panel). Nevertheless, the shape of the curves of the full model and of the universal behavior given by equation (30)-(33) is still very similar at $T=0.05$. This comparison indicates the region of temperatures and fields within which the universal behavior (30)-(33) determined by $x=h / T$ emerges. It is obvious that for larger magnetic fields (i.e., large values of $x$ in Fig. 4) the localized-magnon states cover the thermodynamics not only at very low temperatures.

In Figure 5 we show the specific heat in dependence on $x=h / T$ at the same three temperatures as in Figure 4 for 

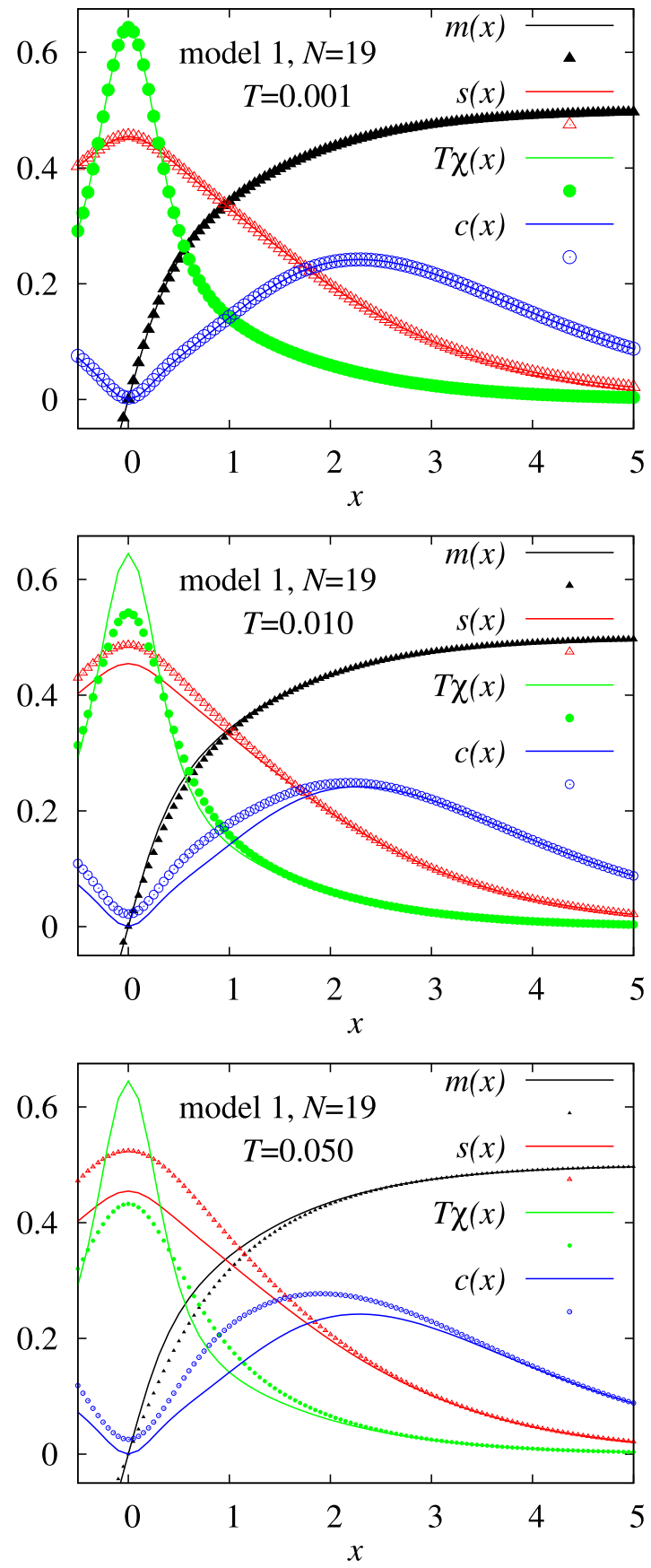

Fig. 4. Universal dependences $m(x), s(x), T \chi(x)$, and $c(x)$ versus $x=h / T$ for the open sawtooth-chain model 1 with $N=$ $19(\mathcal{N}=9)$. Symbols correspond to the full model $\left(J_{1}=1\right)$ at different temperatures $T=0.001,0.01,0.05$ (from top to bottom), lines correspond to formulas (30)-(33).

$N=17$ and $N=19$ (open boundary conditions imposed) and $N=16$ and $N=20$ (periodic boundary conditions imposed). Comparing the data for $N=16$ and $N=20$ as well as for $N=17$ and $N=19$ at $T=0.001$ (black curves) it is evident that the finite-size effects are small (finite-size effects cannot be seen in this scale for $T=0.01$ and $T=0.05$ and therefore the data for $N=16,17$ are not shown at these temperatures). However, comparing,

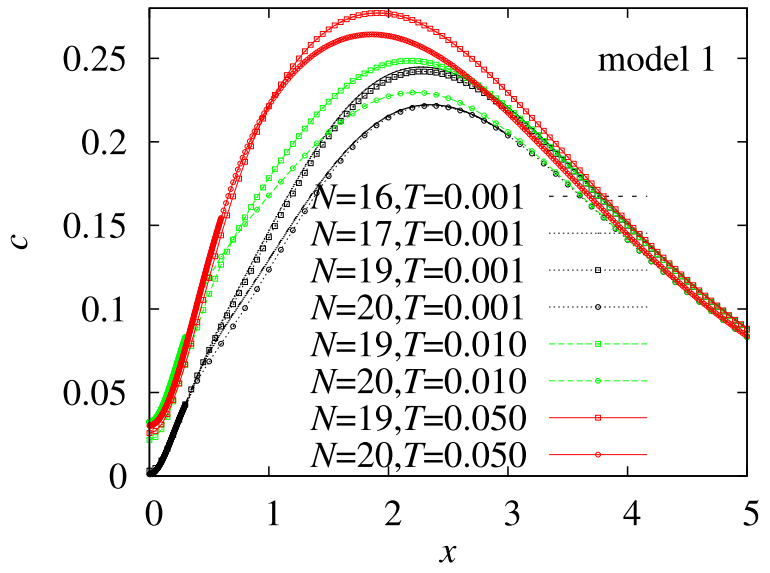

Fig. 5. Specific heat versus $x=h / T$ at low temperatures $T=0.001$ (black), $T=0.01$ (green), $T=0.05$ (red) for the sawtooth-chain model $1\left(J_{1}=1\right)$ of $N=16,17,19,20$ sites.

e.g., $N=19$ with $N=20$ there is a noticeable influence of the boundary conditions for the considered finite values of $N$.

As already mentioned above, the universal behavior is identical for models $\mathbf{1}$ and $\mathbf{2}$ (but not for model $\mathbf{3}$ ). Leaving the range of validity of formulas (30)-(33) which display the contributions of flat-band states only, the physics of the full spin model is determined more and more also by the non-flat-band states. This issue is studied in the next section by large-scale numerical calculations of the full spin models $\mathbf{1}, \mathbf{2}$, and $\mathbf{3}$.

\section{Numerical investigations of finite systems}

Now we consider models $\mathbf{1}$ and $\mathbf{2}$ at zero field and model $\mathbf{3}$ at the saturation field, i.e., all excitations are non-flatband states. We present full exact diagonalization (ED) and finite-temperature Lanczos (FTL) data for the considered sawtooth-chain models of finite size $N$. We focus here on periodic chains allowing to access larger system-sizes $N$ by exploiting the translational symmetry not present in open chains. Using ED we can study up to $N=20$ and using FTL we will provide data up to $N=32$.

We begin with the excitation gaps $\Delta^{(i)}\left(S^{z}\right)(i=1$ corresponds to model $\mathbf{1}$ and $i=2$ corresponds to model $\mathbf{2}$ ), considered in each sector of $S^{z}$ separately, see Table 1 for $N=20$ (and also Tab. I in Ref. [16] for model $\mathbf{2}$ with $N=16,20,24,28$ and $k=1, \ldots, 6)$. For both models the gaps $\Delta^{(i)}\left(S^{z}=N / 2-k\right)$ are rather small if $k>1$. However, for model 2 the gap $\Delta^{(2)}$ becomes virtually zero for $k>4$, but not for model $\mathbf{1}$. This means that the contribution of low-lying excited states to the partition function enters for model $\mathbf{2}$ at lower temperatures than for model $\mathbf{1}$, compare the specific heat in the low-temperature region shown in Figure 7 and discussed below.

First, we pass to the density of states, see Figure 6 . Although the models $\mathbf{1}\left(J_{1}=1\right.$, blue curves) and $\mathbf{2}\left(J_{1}=\right.$ $1 / 2$, magenta curves) do not have identical energy spectra, a similarity between both models is evident. On the other hand, the density of states of model $3\left(J_{1}=1 / 2\right.$, red 
Table 1. Ground-state degeneracies $G^{(i)}\left(S^{z}\right)$ and excitation gaps $\Delta^{(i)}\left(S^{z}\right) \equiv E_{1}^{(i)}\left(S^{z}\right)-E_{0}$ for the periodic sawtooth-chain model $\mathbf{1}\left(J_{1}=1, i=1\right)$ and model $\mathbf{2}$ $\left(J_{1}=1 / 2, i=2\right)$ both of $N=20$ sites $(\mathcal{N}=10)$ in different subspaces $S^{z} . E_{1}^{(i)}\left(S^{z}\right)$ is the energy of the lowest excitation in the subspace $S^{z}$ and $E_{0}=-3.75$ is the ground-state energy (which is identical for models $\mathbf{1}$ and 2). The results in the third and fifth columns coincide with the predictions according to equations (27) and (26). For (finite) periodic sawtooth chains of $N=20$ sites, the total number of ground states for model $\mathbf{1}$ is 4445 and for model $\mathbf{2}$ it is 3545 .

\begin{tabular}{llllll}
\hline$S^{z}$ & $k$ & $G^{(1)}\left(S^{z}\right)$ & $\Delta^{(1)}\left(S^{z}\right)$ & $G^{(2)}\left(S^{z}\right)$ & $\Delta^{(2)}\left(S^{z}\right)$ \\
\hline 9 & 1 & 10 & 0.5 & 10 & 1.0 \\
8 & 2 & 45 & 0.0269961100 & 45 & 0.0217767454 \\
7 & 3 & 121 & 0.0112132000 & 120 & 0.0004848763 \\
6 & 4 & 220 & 0.0058587800 & 210 & 0.0000132138 \\
5 & 5 & 297 & 0.0021102500 & 252 & 0.0000001974 \\
4 & 6 & 332 & 0.0022560900 & 252 & 0.0000000641 \\
3 & 7 & 341 & 0.0031163200 & 252 & 0.0000000641 \\
2 & 8 & 342 & 0.0038286200 & 252 & 0.0000000358 \\
1 & 9 & 342 & 0.0032479000 & 252 & 0.0000000075 \\
0 & 10 & 343 & 0.0037928600 & 253 & 0.0000000075 \\
\hline
\end{tabular}

curves in Fig. 6) is completely different. A striking feature of the density of states of models $\mathbf{1}$ and $\mathbf{2}$ is the collection of about $6 \%$ of the states in the low-energy region below $E-E_{0} \lesssim 0.6$, where this region is separated by a quasi-gap from the high-energy region $E-E_{0} \gtrsim 0.6$. This feature together with the huge ground-state degeneracy is responsible for the unconventional low-temperature physics of models $\mathbf{1}$ and $\mathbf{2}$.

We have to comment on the height of the blue and magenta peaks at $E-E_{0}=0$ in Figure 6 . As it has been explained above, the ground-state degeneracy for the periodic model 1 (4445) is larger than for the periodic model 2 (3545), see Table 1 . That would imply that the blue peak at $E-E_{0}=0$ is higher than the magenta one. However, the gaps for model $\mathbf{2}$ are much smaller than for model 1 and within the first histogram bar between $E_{0}$ and $E_{0}+\Delta E$ with $\Delta E=0.02$ or $\Delta E=0.002$ not only the ground states but also excited states are collected. According to the above discussion of the gaps, there are a lot of excited states in the first $\Delta E$ interval for model $\mathbf{2}$ but much less for model $\mathbf{1}$.

Finally, in Figure 7 we show the temperature dependence (logarithmic temperature scale) of the specific heat for all three models (models $\mathbf{1}$ and $\mathbf{2}$ at zero field and model $\mathbf{3}$ at the saturation field). The specific heat of model $\mathbf{3}$ is characterized by single pronounced maximum followed by an exponential decay of $c(T)$ as $T \rightarrow 0$ leading to a virtually vanishing specific heat below $T \sim 0.06$. By contrast, the very specific low-energy density of states of models $\mathbf{1}$ and $\mathbf{2}$ with much smaller energy gaps and the quasigap at about $E=E_{0}+0.06$ leads to a distinct separation of temperature scales in the temperature dependence of the specific heat which is characterized by a pronounced
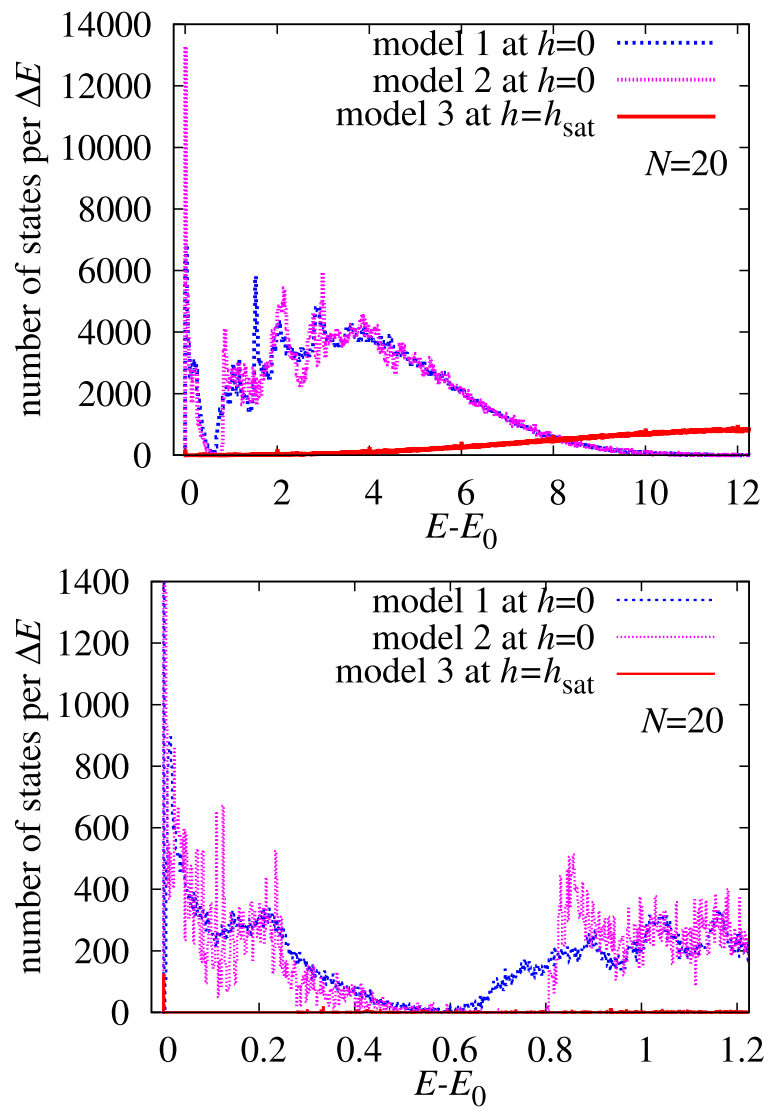

Fig. 6. Density of states (histogram) for the models $\mathbf{1}$ and $\mathbf{2}$ at $h=0$ (blue and magenta) and for model $\mathbf{3}$ at $h=h_{\text {sat }}$ (red) for periodic chains of $N=20$ sites (ED). Top: histogram bar width $\Delta E=0.02$. Bottom: histogram bar width $\Delta E=0.002$, only low-energy part, where the $y$-axis is cut at 1400 to improve the visibility.

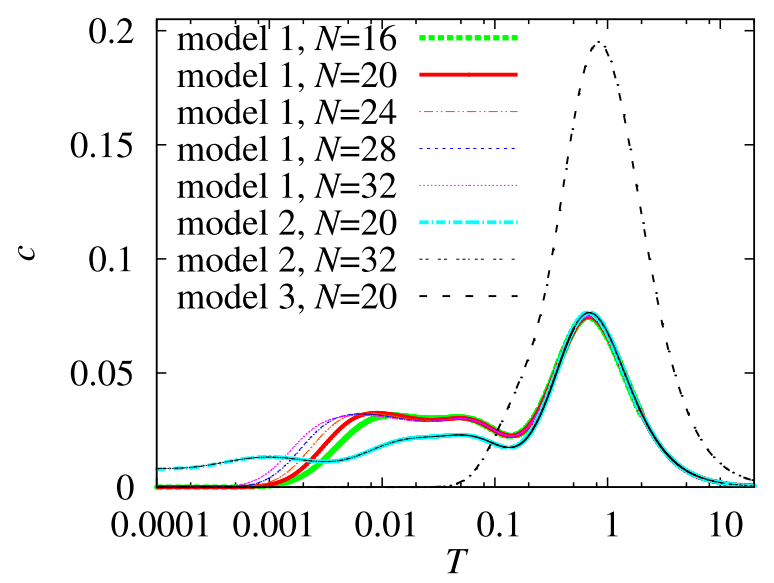

Fig. 7. Specific heat (logarithmic temperature scale) for periodic model $\mathbf{1}\left(J_{1}=1\right)$ and model $\mathbf{2}\left(J_{1}=1 / 2\right)$ at $h=0$ as well as for periodic model $\mathbf{3}\left(J_{1}=1 / 2\right)$ at $h=h_{\text {sat }}$ $\left(h_{\text {sat }}=2\right)$. Exact diagonalization data $(N=16,20)$ and finitetemperature Lanczos data $(N=24,28,32 ; R=20$ for model 1 and $R=10$ for model 2). Finite-size effects are small. 


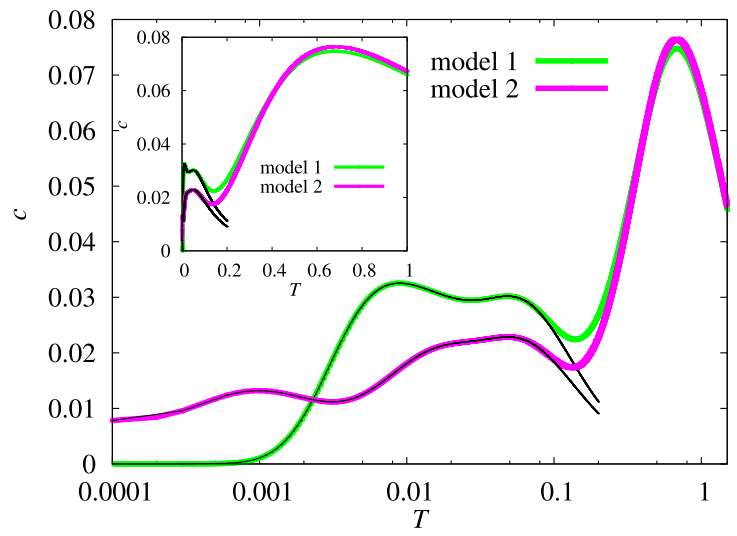

Fig. 8. Specific heat $c(T)$ (exact diagonalization data, periodic chain $N=20)$ for model $1\left(J_{1}=1\right)$ and model $\mathbf{2}\left(J_{1}=1 / 2\right)$ at $h=0$ : green and magenta lines - exact data using the full spectrum, black lines - approximate data using only the lowenergy spectrum below the quasi-gap. Main panel: logarithmic temperature scale. Inset: linear temperature scale.

low-temperature profile of $c(T)$ with two additional maxima below the typical main maximum. The difference in the details of the low-energy spectrum of the two models results in a deviation of the $c(T)$ curves of both models at low temperatures starting at about $T=0.3$. For model 1 the finite-size effects are negligible down to $T \sim 0.01$, whereas model $\mathbf{2}$ exhibits practically no finite-size effects in the temperature region shown in Figure 7. This difference can be attributed to the different sizes of the excitation gaps, cf. Table 1.

To demonstrate the relation of the separation of temperature scales in the $c(T)$ profile to the very specific structure of the density of states of the models $\mathbf{1}$ and $\mathbf{2}$ we show in Figure 8 the specific heat $c(T)$ for periodic chains of $N=20$ sites using the full spectrum (i.e., numerically exact data) together with the approximate data for $c(T)$ which are calculated using a restricted set of energies $E<E_{0}+0.06$, i.e., only the low-energy spectrum below the quasi-gap is taken into account. This comparison reveals that indeed the unconventional features in $c(T)$ below the main maximum are entirely covered by the energy levels below the quasi-gap.

\section{Conclusions}

To summarize, in the present paper we have examined three spin-1/2 sawtooth-chain models which for a special choice of parameters exhibit flat-band physics. While the universal flat-band behavior of the two models introduced in references [25] and [16] is identical, it is different from the universal flat-band behavior of the model introduced in references [5,13]: In the latter case flat-band many-magnon localized states are independent localized magnons only, whereas in the former two cases the flat-band ground-state manifold is larger containing in addition specifically overlapping localized magnons (many-magnon complexes). Flat-band states dominate the low-temperature thermodynamics around zero field (models $\mathbf{1}$ and $\mathbf{2}$ ) or around the saturation field (model $\mathbf{3}$ ). The localized nature of flat-band states allows the complete analysis of the massively degenerate ground-state manifold, and, as a result, to find analytical expressions for the low-temperature thermodynamics in presence of a magnetic field. These analytical expressions are complemented by numerical calculations of thermodynamic quantities of finite sawtooth spin chains.

The spin-1/2 $X X Z 0$ sawtooth-chain model admits another promising route to examine its properties using the three-coloring representation [25,26]. Interesting and still open problems are: how to present the localized many-magnon complex states within the three-coloring picture? Is it possible to use the three-coloring representation for constructing thermodynamics? In the future, it might be interesting to consider small perturbations to the basic flat-band sawtooth-chain models (1). One extension is to introduce into Hamiltonian (1) the interaction between neighboring apical sites $J_{1}^{\prime}, \Delta_{1}^{\prime}$ to see traces of the flat-band-states manifolds.

Open access funding provided by Projekt DEAL. O. D. acknowledges the kind hospitality of the MPIPKS, Dresden in September-November of 2019. This work was supported by the Deutsche Forschungsgemeinschaft DFG (314331397 (SCHN 615/23-1); 355031190 (FOR 2692); 397300368 (SCHN 615/251)). Computing time at the Leibniz Center in Garching is gratefully acknowledged.

\section{Author contribution statement}

All the authors were involved in the preparation of the manuscript. All the authors have read and approved the final manuscript.

Open Access This is an open access article distributed under the terms of the Creative Commons Attribution License (http://creativecommons.org/licenses/by/4.0), which permits unrestricted use, distribution, and reproduction in any medium, provided the original work is properly cited.

Publisher's Note The EPJ Publishers remain neutral with regard to jurisdictional claims in published maps and institutional affiliations.

\section{Appendix A: Some analytical calculations supplementary to Section 3}

Here we present some detailed analytical calculations mentioned but not presented in the main text.

First we check that in the subspace $S^{z}=N / 2-1$ the boundary and bulk localized-magnon states defined in equations (14) and (15) are exact ground states for model 1 of $N=2 \mathcal{N}+1$ sites with open boundary conditions, where $\mathcal{N}$ is the number of triangles in this chain. We begin with the boundary state

$$
\left|l_{1}\right\rangle=\left(-\left|\downarrow_{1} \uparrow_{2} \uparrow_{3}\right\rangle+\left|\uparrow_{1} \downarrow_{2} \uparrow_{3}\right\rangle\right)|\ldots \uparrow \ldots\rangle .
$$


That is a state with one flipped spin located on the boundary bond $J_{2}$ of the zig-zag path, cf. Figure 1, top. This state contains the eigenstate $|2\rangle$ of the Hamiltonian of the first triangle $H_{123}$ with the energy $-3 / 8$, cf. equation (12), and the Hamiltonians of all other triangles act on the fully polarized state $|1\rangle$ (10) giving each time again the energy $-3 / 8$. Therefore, the state $\left|l_{1}\right\rangle$ (A.1) belongs to the ground-state manifold of model 1 with the energy $(-3 / 8) \mathcal{N}$. We pass to the bulk state

$$
\left|l_{2}\right\rangle=\left(\left|\downarrow_{2} \uparrow_{3} \uparrow_{4}\right\rangle-\left|\uparrow_{2} \downarrow_{3} \uparrow_{4}\right\rangle+\left|\uparrow_{2} \uparrow_{3} \downarrow_{4}\right\rangle\right)|\ldots \uparrow \ldots\rangle,
$$

where the numbers at the up- and down-arrows correspond to the numbering in Figure 1, top. We consider the application of $H_{123}$ and $H_{345}$ on $\left|l_{2}\right\rangle$ (A.2) (all Hamiltonians $H_{\triangle}$ of other triangles act on the fully polarized state $|1\rangle_{\triangle}$ yielding the energy $\left.-3 / 8\right)$. We notice that $H_{123}$ acts either on $\left(\left|\uparrow_{1} \downarrow_{2} \uparrow_{3}\right\rangle-\left|\uparrow_{1} \uparrow_{2} \downarrow_{3}\right\rangle\right) \mid \ldots \uparrow$ $\ldots\rangle=(|3\rangle-|2\rangle)_{123}|\ldots \uparrow \ldots\rangle$ or on $\left|\uparrow_{1} \uparrow_{2} \uparrow_{3}\right\rangle|\ldots\rangle=$ $|1\rangle_{123}|\ldots\rangle$, i.e., on the eigenstates with the energy $-3 / 8$, see equations (12) and (10) in Section 2.2. Furthermore, $H_{345}$ acts either on $\left|\uparrow_{3} \uparrow_{4} \uparrow_{5}\right\rangle|\ldots\rangle=|1\rangle_{345}|\ldots\rangle$ or on $\left(-\left|\downarrow_{3} \uparrow_{4} \uparrow_{5}\right\rangle+\left|\uparrow_{3} \downarrow_{4} \uparrow_{5}\right\rangle\right)|\ldots \uparrow \ldots\rangle=(-|2\rangle)_{345}|\ldots \uparrow \ldots\rangle$, i.e., once more on the eigenstates with the energy $-3 / 8$. Hence, the state $\left|l_{2}\right\rangle$ (A.2) also belongs to the ground-state manifold of model $\mathbf{1}$.

Next we check that overlapping localized two-magnon complexes, equations (17) and (18), are eigenstates of the Hamiltonian (2) with the eigenvalue $(-3 / 8) \mathcal{N}$. We start with the state (17) and consider first how the Hamiltonian $H_{123}$ acts on this state. It acts either on $\left(-s_{1}^{-}+s_{2}^{-}\right)\left(s_{1}^{-} / 2+s_{2}^{-} / 2-s_{3}^{-}\right)|0\rangle=(-|5\rangle)_{123} \mid \ldots \uparrow$ $\ldots\rangle$ or on $\left(-s_{1}^{-}+s_{2}^{-}\right) s_{4}^{-}|0\rangle=(-|2\rangle)_{123}|\ldots\rangle$. In both cases, $H_{123}$ acts on its eigenstates with the energy $-3 / 8$. Next, $H_{345}$ acts either on $\left|\uparrow_{3} \uparrow_{4} \uparrow_{5}\right\rangle|\ldots\rangle=|1\rangle_{345}|\ldots\rangle$ or on $\left(-s_{3}^{-}+s_{4}^{-}\right) \ldots|0\rangle=(-|2\rangle)_{345}|\ldots\rangle$, i.e., on its eigenstates with the energy $-3 / 8$. Finally, all other $H_{\triangle}$ acts on $|1\rangle_{\triangle}|\ldots\rangle$, i.e., on their eigenstates with the energy $-3 / 8$. As a result, we conclude that the localized two-magnon complex $l_{1}\left(c l_{1}+l_{2}\right)|0\rangle(17)$ is indeed among the ground states with $S^{z}=N / 2-2$.

Now we pass to the state (18). We have to consider the application of $H_{123}, H_{345}$, and $H_{567}$ on the state (18), since the rest Hamiltonians $H_{\triangle}$, while acting on the state (18), "see" the only relevant factor $|1\rangle_{\triangle}$ which is the eigenstate (10) with the eigenvalue $-3 / 8$. We begin with $H_{123}$. It acts either on $\left(s_{2}^{-}-s_{3}^{-}\right)\left(-s_{1}^{-}+s_{2}^{-} / 2+s_{3}^{-} / 2\right)|0\rangle=(|6\rangle-|5\rangle)_{123} \mid \ldots \uparrow$ $\ldots\rangle$, or on $\left(s_{2}^{-}-s_{3}^{-}\right) \ldots|0\rangle=(|3\rangle-|2\rangle)_{123}|\ldots\rangle$, or on $\left(-s_{1}^{-}+s_{2}^{-} / 2+s_{3}^{-} / 2\right) \ldots|0\rangle=(-|2\rangle / 2-|3\rangle / 2)_{123}|\ldots\rangle$, or on $|1\rangle_{123}|\ldots\rangle$, i.e., each time on the eigenstates with the eigenvalue $-3 / 8$. Next, $H_{345}$ acts either on $|1\rangle_{345}|\ldots\rangle$, or on $\left(s_{3}^{-} / 2+s_{4}^{-} / 2-s_{5}^{-}\right) \ldots|0\rangle=(|3\rangle-|2\rangle / 2)_{123}|\ldots\rangle$, or on $\left(-s_{3}^{-}+s_{4}^{-}\right) \ldots|0\rangle=(-|2\rangle)_{345}|\ldots\rangle$, or on $\left(-s_{3}^{-}+s_{4}^{-}\right)\left(s_{3}^{-} / 2+s_{4}^{-} / 2-s_{5}^{-}\right)|0\rangle=(-|5\rangle)_{345} \mid \ldots \uparrow$ ...), i.e., each time on the eigenstates with the eigenvalue $-3 / 8$. Finally, $H_{567}$ acts either on $|1\rangle_{567}|\ldots\rangle$ or on $\left(-s_{5}^{-}+s_{6}^{-}\right) \ldots|0\rangle=(-|2\rangle)_{567}|\ldots\rangle$, i.e., each time on the eigenstates with the eigenvalue $-3 / 8$. In sum, the state $l_{2}\left(l_{1}+c l_{2}+l_{3}\right)|0\rangle$ (18) belongs to the ground-state manifold with $S^{z}=N / 2-2$. Similar calculations for the states $l_{3}\left(l_{2}+c l_{3}+l_{4}\right)|0\rangle, \ldots, l_{\mathcal{N}+1}\left(l_{\mathcal{N}}+c l_{\mathcal{N}+1}\right)|0\rangle$ confirm that this kind of states belongs to the ground-state manifold with $S^{z}=N / 2-2$.

We consider now a localized three-magnon complex of the one-bracket type given in equation (20). In more detail it reads

$$
\begin{aligned}
l_{2} l_{4} & \left(l_{1}+c l_{2}+l_{3}+c l_{4}+l_{5}\right)|0\rangle \\
= & \left(s_{2}^{-}-s_{3}^{-}+s_{4}^{-}\right)\left(s_{6}^{-}-s_{7}^{-}+s_{8}^{-}\right) \\
& \times\left(-s_{1}^{-}+\frac{s_{2}^{-}}{2}+\frac{s_{3}^{-}}{2}+\frac{s_{4}^{-}}{2}-s_{5}^{-}+\frac{s_{6}^{-}}{2}+\frac{s_{7}^{-}}{2}+\frac{s_{8}^{-}}{2}-s_{9}^{-}+s_{10}^{-}\right)|0\rangle
\end{aligned}
$$

$(c=-1 / 2)$, see line 3 in Figure 3 . We have to check whether this state is an eigenstate of the Hamiltonians $H_{123}, H_{345}, H_{567}, H_{789}$, and $H_{9,10,11}$ with the eigenvalue $-3 / 8$. As explained above, the Hamiltonians $H_{123}$ and $H_{567}$ while acting on the state (A.3) "see" only their eigenstates $|6\rangle-|5\rangle,|3\rangle-|2\rangle,-|2\rangle / 2-|3\rangle / 2$, and $|1\rangle$; all with the eigenvalue $-3 / 8$. Next, the Hamiltonians $H_{345}$ and $H_{789}$ while acting on the state (A.3) "see" only their eigenstates $-|5\rangle,-|2\rangle,-|2\rangle / 2+|3\rangle$, and $|1\rangle$; all with the eigenvalue $-3 / 8$. Finally, the Hamiltonian $H_{9,10,11}$ while acting on the state (A.3) "sees" only its eigenstates $-|2\rangle_{9,10,11}$ and $|1\rangle_{9,10,11}$; both with the eigenvalue $-3 / 8$. Hence, the state given in equation (A.3) [or Eq. (20)] belongs to the ground-state manifold with $S^{z}=N / 2-3$.

Now we check that localized three-magnon complexes of the two-bracket type are ground states. We consider, for example, the state given in the third line of equation (22), i.e.,

$$
\begin{aligned}
& {\left[\left(s_{4}^{-}-s_{5}^{-}+s_{6}^{-}\right)\left(s_{2}^{-}-s_{3}^{-}+\frac{s_{4}^{-}}{2}+\frac{s_{5}^{-}}{2}+\frac{s_{6}^{-}}{2}-s_{7}^{-}+s_{8}^{-}\right)\right.} \\
& \times\left(-s_{1}^{-}+\frac{s_{2}^{-}}{2}+\frac{s_{3}^{-}}{2}-\frac{s_{4}^{-}}{4}-\frac{s_{5}^{-}}{4}-\frac{s_{6}^{-}}{4}+\frac{s_{7}^{-}}{2}+\frac{s_{8}^{-}}{2}-s_{9}^{-}+s_{10}^{-}\right) \\
& \left.-\frac{3}{4} s_{4}^{-} s_{5}^{-} s_{6}^{-}\right]|0\rangle,
\end{aligned}
$$

see line 6 in Figure 3. Checking that the Hamiltonians $H_{123}, H_{789}, H_{9,10,11}$ and so on while acting on the state (A.4) give $-3 / 8$ multiplied by this state is straightforward by repetition of the calculations explained above. The role of the terms with $d$ in equation (22) becomes clear after acting on the state (A.4) by the Hamiltonians $H_{345}$ and $H_{567}$ : only after accounting the term $(-3 / 4) s_{4}^{-} s_{5}^{-} s_{6}^{-}$ these Hamiltonians "see" some linear combinations of their eigenstates with the eigenvalue $-3 / 8$. As a result, in all cases we arrive at the state (A.4) multiplied by $-3 / 8$. Hence the state (A.4) is within the ground-state manifold with $S^{z}=N / 2-3$. 


\section{References}

1. O. Derzhko, J. Richter, M. Maksymenko, Int. J. Mod. Phys. B 29, 1530007 (2015)

2. M.E. Zhitomirsky, H. Tsunetsugu, Phys. Rev. B 70, 100403(R) (2004)

3. O. Derzhko, J. Richter, Phys. Rev. B 70, 104415 (2004)

4. M.E. Zhitomirsky, H. Tsunetsugu, Prog. Theor. Phys. Suppl. 160, 361 (2005)

5. O. Derzhko, J. Richter, Eur. Phys. J. B 52, 23 (2006)

6. M.E. Zhitomirsky, H. Tsunetsugu, Phys. Rev. B 75, 224416 (2007)

7. J. Schnack, J. Schulenburg, J. Richter, Phys. Rev. B 98, 094423 (2018)

8. A. Mielke, J. Phys. A 24, L73 (1991)

9. A. Mielke, J. Phys. A 24, 3311 (1991)

10. H. Tasaki, Phys. Rev. Lett. 69, 1608 (1992)

11. O. Derzhko, J. Richter, A. Honecker, M. Maksymenko, R. Moessner, Phys. Rev. B 81, 014421 (2010)

12. M. Maksymenko, A. Honecker, R. Moessner, J. Richter, O. Derzhko, Phys. Rev. Lett. 109, 096404 (2012)

13. J. Schulenburg, A. Honecker, J. Schnack, J. Richter, H.-J. Schmidt, Phys. Rev. Lett. 88, 167207 (2002)

14. J. Richter, O. Derzhko, A. Honecker, Int. J. Mod. Phys. B 22, 4418 (2008)

15. A. Metavitsiadis, C. Psaroudaki, W. Brenig, Phys. Rev. B 101, 235143 (2020)

16. V.Y. Krivnov, D.V. Dmitriev, S. Nishimoto, S.-L. Drechsler, J. Richter, Phys. Rev. B 90, 014441 (2014)

17. T. Tonegawa, M. Kaburagi, J. Magn. Magn. Mater. 272276, 898 (2004)

18. M. Kaburagi, T. Tonegawa, M. Kang, J. Appl. Phys. 97, 10B306 (2005)

19. D.V. Dmitriev, V.Y. Krivnov, Phys. Rev. B 92, 184422 (2015)

20. D.V. Dmitriev, V.Y. Krivnov, J. Phys.: Condens. Matter 29, $215801(2017)$

21. D.V. Dmitriev, V.Y. Krivnov, J. Richter, J. Schnack, Phys. Rev. B 99, 094410 (2019)
22. D.V. Dmitriev, V.Y. Krivnov, J. Schnack, J. Richter, Phys. Rev. B 101, 054427 (2020)

23. A. Baniodeh, N. Magnani, Y. Lan, G. Buth, C.E. Anson, J. Richter, M. Affronte, J. Schnack, A.K. Powell, Quantum Mater. 3, 10 (2018)

24. R. Shirakami, H. Ueda, H.O. Jeschke, H. Nakano, S. Kobayashi, A. Matsuo, T. Sakai, N. Katayama, H. Sawa, K. Kindo, C. Michioka, K. Yoshimura, Phys. Rev. B 100, 174401 (2019)

25. H.J. Changlani, D. Kochkov, K. Kumar, B.K. Clark, E. Fradkin, Phys. Rev. Lett. 120, 117202 (2018)

26. H.J. Changlani, S. Pujari, C.-M. Chung, B.K. Clark, Phys. Rev. B 99, 104433 (2019)

27. M. Axenovich, M. Luban, Phys. Rev. B 63, 100407(R) (2001)

28. O. Cepas, A. Ralko, Phys. Rev. B 84, 020413(R) (2011)

29. K. Essafi, O. Benton, L.D.C. Jaubert, Nat. Commun. 7, 10297 (2016)

30. J. Richter, J. Schulenburg, Eur. Phys. J. B 73, 117 (2010)

31. J. Jaklič, P. Prelovšek, Phys. Rev. B 49, 5065(R) (1994)

32. J. Jaklič, P. Prelovšek, Adv. Phys. 49, 1 (2000)

33. B. Schmidt, P. Thalmeier, Phys. Rep. 703, 1 (2017)

34. J. Schnack, O. Wendland, Eur. Phys. J. B 78, 535 (2010)

35. J. Schnack, J. Richter, R. Steinigeweg, Phys. Rev. Research 2, 013186 (2020)

36. J.M. Radcliffe, J. Phys. A Gen. Phys. 4, 313 (1971)

37. H.-J. Schmidt, J. Richter, R. Moessner, J. Phys. A 39, 10673 (2006)

38. M.E. Zhitomirsky, A. Honecker, J. Stat. Mech. Theor. Exp. 2004, P07012 (2004)

39. J. Schnack, R. Schmidt, J. Richter, Phys. Rev. B 76, 054413 (2007)

40. M. Evangelisti, E.K. Brechin, Dalton Trans. 39, 4672 (2010)

41. E. Garlatti, S. Carretta, J. Schnack, G. Amoretti, P. Santini, Appl. Phys. Lett. 103, 202410 (2013) 